\title{
Conocimiento y Uso de las Tecnologías de la Información y las Comunicaciones (TIC) según el Estilo de Aprendizaje de los Futuros Maestros
}

\author{
Ramón Cózar-Gutiérrez ${ }^{(1)}$, María V. De Moya-Martínez ${ }^{(2)}$, José A., Hernández-Bravoo ${ }^{(2)}$ y \\ Juan R., Hernández-Bravo ${ }^{(2)}$ \\ (1) Universidad Castilla La Mancha, Departamento de Historia. Facultad Educación, Plaza Universidad, 3 \\ (02006) Albacete - España. (e-mail: ramon.cozar@uclm.es) \\ (2) Universidad Castilla La Mancha. Departamento Didáctica de la Expresión Musical, Plástica y Corporal. \\ Facultad Educación, Plaza Universidad, 3 (02006) Albacete - España. \\ (e-mail: mariavallede.moya@uclm.es; josea.hernandez@uclm.es; juanrafael.hernandez@uclm.es)
}

Recibido Jun. 2, 2016; Aceptado Ago. 1, 2016; Versión final Sep. 13, 2016, Publicado Dic. 2016

\begin{abstract}
Resumen
El presente trabajo de investigación ha tenido como principal objetivo determinar el grado de conocimiento, uso y valoración de las Tecnologías de la Información y el Conocimiento (TIC) entre el alumnado de los Grados de Maestro de la Facultad de Educación de Albacete (Universidad de Castilla-La Mancha, España). Esto con el fin de evaluar, en investigaciones posteriores, su adquisición de la competencia digital. El instrumento utilizado fue el cuestionario REATIC, administrado a alumnos de las titulaciones de Maestro en Educación Infantil y Primaria $(n=162)$, centrado en el manejo y aceptación de las nuevas tecnologías y su relación con el estilo de aprendizaje predominante del usuario. Los resultados demostraron que los estudiantes poseen un alto grado de conocimientos y usos de herramientas tecnológicas, además de una ligera predominancia de los estilos pragmático y teórico. Consideramos que son factores a tener en cuenta por el profesorado universitario, preocupado en ofrecer una enseñanza de calidad acorde con nuestros tiempos.
\end{abstract}

Palabras clave: TIC; estilos de aprendizaje; educación superior; estudiantes; grado de maestro

\section{Knowledge and use of Information and Communications Technology (ICT) by Prospective Teachers According to their Learning Styles}

\begin{abstract}
The current research aims to study the relationship among knowledge, use and appreciation of Information and Communications Technology (ICT) in students of Bachelor Degrees in Early Childhood and Primary Education at the Faculty of Education in Albacete (University of Castilla La Mancha, Spain). This was done with the objective of evaluating, in forthcoming research projects, the digital competence of the students. The instrument used was the REATIC questionnaire, which was administered to students of Bachelor Degree in Teacher of Early Childhood and Primary Education $(n=162)$ to evaluate the knowledge and use of new technologies and the relationship between ICT and student's learning styles. Results showed a high degree of knowledge and use of ICT, as well as a slight predominance of pragmatic and theoretical styles, facts to be considered by professors, in order to offer the best quality in education according to our technological age.
\end{abstract}




\section{INTRODUCCIÓN}

La época de aceleración y cambios que vivimos afecta, como no podía ser de otra manera, a la educación. Así, las aulas demandan nuevas necesidades formativas, por parte del profesor, y una adaptación tecnológica que vaya acorde con la realidad imperante y las costumbres que se han apoderado de una sociedad, haciendo que ésta sea cada vez más virtual. Destacar con cierta profundidad las múltiples posibilidades que las tecnologías ofrecen para el aprendizaje, excede la tarea de un artículo. Baste indicar que su accesibilidad, interactividad y flexibilidad las convierte en aliados poderosos de las nuevas estrategias de aprendizaje, especialmente en la educación superior.

En nuestro caso, como docentes de la Facultad de Educación, centro en el que se forman los futuros maestros, es preceptivo y obligado incluir las TIC en nuestros procesos de enseñanza, facilitando a nuestros alumnos que mejoren sus aprendizajes, ayudándoles a adquirir unas habilidades específicas que contribuyan a que sean digitalmente competentes. Es decir, que aprendan a adquirir conocimientos, destrezas y actitudes que les capaciten para saber hacer, desarrollar aprendizajes significativos y funcionales y resolver problemas desde una óptica innovadora y creativa. La capacidad motivadora que ejercen las TIC como herramienta de aprendizaje puede fluctuar o verse influenciada por los estilos de aprendizajes predominantes de los discentes (García-Cué et al., 2009).

Fruto de la preocupación de los docentes por incluir las TIC en su labor profesional, ha sido la proliferación de una abundante y valiosa bibliografía sobre el tema (Cabero, 2014; Galanouli et al., 2004; Gallego et al., 2010; Kirschner y Davis, 2003; Llorente, 2008; Mcvee et al., 2008; Morón et al., 2015; Prendes et al., 2010; Rangel, 2015; Raposo et al., 2006; Silva et al., 2006; Tello y Aguaded, 2009; Tondeur et al., 2007; Yuen et al., 2008), en los que se coincide en la necesidad de acometer una "realfabetización digital compensatoria" del profesorado (Gutiérrez, 2008), imponiéndose, en el momento actual, la tendencia que integra la triple formación docente en los ámbitos de lo disciplinar, lo pedagógico y lo tecnológico, siguiendo el modelo TPACK (Technological Pedagogical Content Knowledge, de Koehler y Mishra (2008). Junto a esta línea de investigación, preocupan también los intereses de los alumnos hacia las TIC, analizando especialmente la valoración y uso del alumnado de los Grados de Maestro hacia las aplicaciones didácticas de las diferentes herramientas tecnológicas (Cózar y Roblizo, 2014, Roblizo y Cózar, 2015, Roblizo et al., 2015); y el género, como una variable significativa en relación con una segunda o tercera brecha digital (Castaño, 2008; Castaño, et al., 2011; Vázquez y Castaño, 2011), determinada no ya por el acceso, sino por el nivel de einclusión, en la que se hace patente una actitud menos favorable de las mujeres en habilidades y autonomía de uso de las TIC.

A pesar de la globalización virtual existente, de la omnipresencia tecnológica que se ha impuesto en la vida cotidiana de millones de personas, de la floreciente industria y economía de mercado generado en torno a las tecnologías, la realidad nos demuestra que no se llevan a la práctica diaria de las aulas en un altísimo porcentaje, independientemente de niveles de estudios, centros y especialidades (Cabero, 2014). Abunda la legislación, estándares y recomendaciones acerca de la importancia de la formación en TIC del profesorado (García-Cué et al., 2009) pero sin estar respaldadas por unas competencias básicas en la formación inicial ni unas líneas claras para alcanzar este objetivo. Consideramos que el profesor debe ser capaz de crear entornos de aprendizaje virtuales personalizados, siendo de gran ayuda el conocer los estilos de aprendizaje de sus alumnos, y el suyo propio, para poder diseñar y aplicar actividades concretas que mejoren dichos aprendizajes. $Y$ ya que en todo este proceso educativo y didáctico no podemos olvidarnos de las TIC es por lo que defendemos la aplicación de herramientas iniciales de diagnóstico tipo el Cuestionario REATIC (De Moya et al., 2011).

\section{Estilos de Aprendizaje.}

Las investigaciones sobre estilos y estrategias de aprendizaje llevadas a cabo durante los últimos veinte años han demostrado sobradamente su utilidad entre la comunidad docente universitaria, independientemente de la materia, especialidad o facultad (Acevedo et al., 2015; Alducin y Vázquez, 2016; Blumen et al., 2011; Evans et al., 2010; González et al., 2000; Komarraju et al., 2011; Peterson et al., 2009). Es decir, resulta muy útil para cualquier profesor universitario, de todas las áreas y campos de investigación, conocer los estilos de aprendizaje de sus alumnos con el ánimo de optimizar al máximo todo tipo de metodologías que quiera llevar a cabo en sus clases. Sin olvidar que conocer los estilos de aprendizaje del alumnado, sea del nivel que sean, ayuda también a la adquisición de las competencias básicas. Según Castejón (1997), casi todo lo que el individuo hace o puede llegar a hacer es consecuencia de su aprendizaje, de ahí que sea tan importante conocer los diversos estilos cognitivos, perceptivos y de aprendizaje de los alumnos. En este sentido, la investigación educativa debe interesarse en profundizar en los mecanismos y procesos de aprendizaje. 
Superar con éxito las tareas es fruto de un proceso de aprendizaje planificado y consciente al enfrentarse al estudio y a las herramientas utilizadas. El alumno siempre ha sido el protagonista de su propio aprendizaje (Juárez et al., 2012), pero es una nueva tarea del profesor, ayudado por el manejo de sus estilos de aprendizaje, convertirlos en estrategas de la adquisición del conocimiento. Ventura (2013) hace referencia a diversas investigaciones realizadas en los últimos años donde se pone de manifiesto que los estudiantes presentan una tendencia hacia un estilo particular de aprendizaje por ser el más acorde a sus características y habilidades personales. García Retana (2013) también recoge una interesante revisión bibliográfica sobre los cuatro estilos de aprendizaje establecidos por Honey-Alonso, manifestando la reconocida validez del CHAEA como instrumento para determinar dichos estilos ya que "éstos constituyen las conductas más abarcadoras del sujeto ante la situación de aprendizaje" (Vivas, 2002, citado en Rodríguez, 2006: 121). El alto grado de validez del citado cuestionario radica en que los estilos de aprendizaje recogen una importante combinación de factores humanos como experiencias previas, motivaciones, rasgos de personalidad, de fisiología, preferencias de canales comunicativos, incluso del nivel de dominio de uno u otro de los hemisferios cerebrales, permitiendo corroborar la individualidad de cada estilo (Rodríguez, 2006).

Esta línea de pensamiento es también defendida por otros autores (Monereo, 2007) que analizan las estrategias de aprendizaje entendidas como procesos conscientes y deliberados de toma de decisiones del alumno al elegir los conocimientos que considera más útiles y necesarios para alcanzar sus objetivos. Por su parte, Gargallo, Suárez y Ferreras (2007) estiman que conocer los estilos y estrategias de aprendizaje es algo necesario para crear un ambiente de aula que propicie capacidades como la indagación, la reflexión, la crítica constructiva, el aprendizaje colaborativo de los alumnos. Resulta clarificador para conocer la esencia y la evolución de los estilos de aprendizaje el artículo de Pantoja, Duque y Correa (2013), donde, tras revisar la literatura existente al respecto, ofrecen una recopilación de los principales estilos de aprendizaje, primero en una ordenación cronológica para mostrar claramente el desarrollo temporal del tema, y después, una segunda catalogación que agrupa y revela las principales relaciones entre los distintos modelos de estilos.

Los resultados obtenidos por García, Escalante, Fernández, Escandón, Mustri y Puga (2000) nos llevan a reflexionar sobre la gran flexibilidad de los estilos dependiendo del contexto y del transcurso del tiempo; es decir, de la madurez personal del individuo. Este hecho juega a favor del docente ya que le posibilita emparejar los estilos de aprendizaje de sus alumnos para fortalecer el proceso educativo, lo que vendría a fortalecer la filosofía pedagógica de que no basta con incorporar las TIC para resolver satisfactoriamente el proceso de aprendizaje (Cabero, 2014), ya que ha de tenerse en cuenta el gran peso que juega el factor humano (García Retama, 2013).

El gran interés alcanzado por el estudio sobre los estilos de aprendizaje, lejos de finalizarlo, ha abierto nuevos horizontes, sobre todo al permitir otros planteamientos sobre la concepción existente entre la inteligencia y el modo de aprender. Queda claro que son procesos individuales, diferentes en cada persona, y que se ven influenciados y condicionados por múltiples factores. Por todo ello, querer saber qué metodología es la más apropiada para cada estilo de aprendizaje propicia la aparición de diversos interrogantes en lugar de ofrecer una respuesta concreta y simple, siendo esta aparente complicación un nuevo estímulo para seguir trabajando en este campo desde otros puntos de vista (Pantoja et al., 2013).

\section{TIC y Estilos de Aprendizaje.}

Es preciso aunar esfuerzos y emprender nuevas investigaciones que ayuden a entender el nuevo y complejo mundo pedagógico generado por las TIC, marcado por la dificultad añadida de ser un ámbito tremendamente cambiante debido a la rapidez con la que crecen y evolucionan las nuevas tecnologías. Las TIC, en sus diferentes formatos, versiones y utilidades, han conquistado, por derecho propio, un protagonismo en la vida cotidiana de gran parte de la población, sobre todo, la juvenil. Sus profundas implicaciones y su fuerte impacto sociológico es tan inmenso que aún no existen suficientes estudios para medirlo en toda su intensidad (Cózar y De Moya, 2013). Los componentes de la presente sociedad del conocimiento precisan un saber, un uso y un dominio adecuados para gestionar debidamente las habilidades propias de la era digital, tanto para desenvolverse en el momento presente como para capacitarse con vistas al futuro. Es decir, tanto para el estudio de hoy como para el trabajo de mañana, los alumnos deben conocer sus capacidades tecnológicas, con sus fortalezas y debilidades, siendo el contexto escolar el espacio adecuado para aprender a ello y alcanzar una mínima competencia digital (Herrera, 2015).

La incorporación progresiva de las TIC en los proceso de enseñanza - aprendizaje en educación superior, ha revelado la necesidad de una formación permanente por parte de todos los agentes implicados, profesores y alumnos, que tenga en cuenta la forma particular de aprender de los estudiantes en un mundo 
tecnológico, cambiante y dinámico. Las posibilidades de las herramientas TIC, en cuanto a interactividad y adaptación a las necesidades educativas de los estudiantes, facilitan los procesos de aprendizaje de acuerdo con los distintos estilos predominantes en el alumnado. La interacción, por lo tanto, de nuevas tecnologías y estilos de aprendizaje es un campo de estudio de gran interés, en el ámbito universitario, ya que un mayor logro y rendimiento académico pasa por una individualización de la enseñanza, en la que las TIC juegan un papel determinante.

Los objetivos de la presente investigación son los siguientes: (i) Determinar el grado de conocimiento y uso de las TIC por parte de nuestro alumnado universitario; ii) Conocer la opinión y valoración de las herramientas TIC en la vida académica y personal de los estudiantes; iii) Analizar la relación entre el uso de las herramientas TIC y el estilo de aprendizaje predominante de los alumnos; y iv) Observar cuánto difieren los estilos de aprendizaje de los alumnos en el uso de las TIC, en función del género y de la titulación de Maestro que cursan.

\section{MÉTODO}

El estudio siguió un enfoque metodológico de tipo cuantitativo y un diseño no-experimental. Con el fin de abordar los objetivos de investigación, se contrastaron los datos mediante estudios descriptivos y correlacionales (media, desviación típica y estadísticos $F$ y p).

La muestra es de tipo no probabilístico y siguió los criterios de accesibilidad y disponibilidad muestral. Estuvo formada por 162 estudiantes, pertenecientes al último curso $\left(4^{\circ}\right)$ de los Grados de Maestro en Educación Infantil (81) y en Ed. Primaria (81) de la Facultad de Educación de Albacete (Universidad de Castilla-La Mancha, España). Se han elegido los alumnos que están finalizando sus estudios y se encuentran a las puertas de ser futuros maestros, al considerar que disponen del suficiente criterio para valorar de manera adecuada cómo han trabajado las TIC a lo largo de su formación académica. La muestra supone el $75 \%$ del universo posible, lo que conlleva el que, con un nivel de confianza del $95 \%$, el margen de error sea de sólo 3.86. Del total, 35 son hombres (21,6\%) y 127 mujeres $(78,4 \%)$, lo que, a pesar de su no homogeneidad, resulta ser una muestra representativa de la disparidad de género que existe en los estudios de Maestro, en los que existe una amplia superioridad de alumnas. El rango de edad va desde los 20 a los 44 años, con una media de 22.68. El 88,5\% tiene edades comprendidas entre los 20 y los 25 años, concentrándose el $44,8 \%$ del total en los 21 años.

El instrumento utilizado fue el Cuestionario REATIC (De Moya et al., 2011). Está compuesto por 60 ítems mediante los cuales se puede establecer el mayor o menor grado de conocimiento, uso y valoración existente hacia las TIC en la etapa de formación superior universitaria. Además, vincula estos aspectos con el estilo de aprendizaje predominante del encuestado. Por tanto, sus objetivos giran en torno a conocer el nivel de conocimientos que los alumnos poseen sobre las tecnologías de la información y la comunicación, su grado de uso, mayor o menor, la utilidad que les conceden en base a sus características propias, así como el estilo de aprendizaje predominante que puede asociarse al uso que hacen de las mismas. Es decir, se busca averiguar si un determinado estilo de aprendizaje condiciona en el alumno su manejo y valoración positiva hacia la implementación de las TIC en su vida académica y personal.

Para ello, los 60 ítems referidos se agrupan en 4 subgrupos: conozco (ítem 1 al 14); uso (ítem del 15 al 28), considero que las TIC (ítems 29 al 44); uso de las TIC según el estilo de aprendizaje predominante (ítem 4560). Este subgrupo 4 (ítems del 45 al 60) sigue la clasificación de Alonso, Gallego y Honey (1994), de la siguiente manera: activo (ítems 47, 53, 55, 58); reflexivo (ítems 48, 52, 56, 59); teórico (ítems 46, 49, 57, 60) y pragmático (ítems $45,50,51,54)$.

Los tres primeros subgrupos se centran en obtener información precisa sobre el conocimiento, uso y actitudes hacia las diversas TIC que el alumnado se encuentra en su vida universitaria y privada, pero en ambas esferas prima la cotidianeidad de estas herramientas tecnológicas. Asimismo, en cada ítem se describen ejemplos concretos para facilitar su entendimiento (Ej. "Conozco: Algunos navegadores web (Explorer, Mozilla, FireFox, Netscape,...)"; "Uso: Programas educativos de autor (Clic, Jclic, Hot Potatoes, Neobook,...)"). Por su parte, el cuarto subgrupo busca verificar la relación entre estilo de aprendizaje predominante y uso de las TIC. El citado cuestionario busca, en primer lugar, verificar los conocimientos sobre TIC que poseen los alumnos; además de conocer el grado de uso que éstos hacen de las mismas y analizar la utilidad que les conceden en su formación académica y en su vida personal. A estos tres grandes objetivos se añaden otros como son determinar el uso que los alumnos hacen de las TIC, en base a su estilo de aprendizaje, y conocer el estilo que predomina en el uso de las TIC entre el alumnado.

Este instrumento fue sometido a análisis de fiabilidad y validez en su diseño original (De Moya et al., 2011), a partir de: validez de constructo, siguiendo el juicio de 10 expertos de diferentes campos de estudio 
relacionados con los ámbitos de las TIC y los estilos de aprendizaje; consistencia interna, con el alfa de Cronbach; y con la realización de una prueba piloto, seleccionando una pequeña muestra para corregir posibles dificultades a la hora de responder el cuestionario. Posteriormente ha sido utilizado en numerosas investigaciones científicas con resultados satisfactorios (Calvache et al., 2013; Cózar et al., 2015; Simón, 2011; Varela y Novo, 2014). Como prueba de consistencia interna en este caso, se ha calculado el Alpha de Cronbach, obteniéndose un coeficiente de 0.906, índice que refleja un alto grado de consistencia interna de los ítems.

Para la recogida de datos se pasó el cuestionario en horario de clase a comienzos del curso académico 2015-16. Se informó a los estudiantes sobre la finalidad de la investigación, indicándoles que el cuestionario era totalmente anónimo e incidiendo en la importancia de responder con sinceridad a todas las preguntas. Se consiguió una tasa de respuesta del 95\%, muy elevada debido a que se les insistió a los alumnos sobre la conveniencia de sus respuestas con la intención de mejorar la implementación de las TIC en su proceso formativo. El tiempo de cumplimentación osciló entre los 20 y 25 minutos.

La investigación se ha realizado teniendo en cuenta los principios éticos de la American Educational Research Association (2011), como seguir un protocolo de actuación previo al estudio, obtener los permisos necesarios para realizar la investigación, comunicar a los participantes la finalidad del estudio, garantizar la privacidad y confidencialidad de las respuestas de los participantes y comunicar las posibles implicaciones de la investigación. Además, se recoge la información de manera objetiva sin interferir en las respuestas o emitir juicios de valor. Los datos cuantitativos han sido analizados mediante el paquete de programas estadísticos SPSS, versión 19 para Windows. En este sentido, se hallaron estadísticos descriptivos (media y desviación típica) y se comparó la diferencia de medias entre los grupos estudiados (Infantil y Primaria y masculino y femenino) con la prueba ANOVA, estableciendo un nivel de significación de 0,05.

\section{RESULTADOS}

En las tablas 1 a 5 se recogen las percepciones del alumnado de los Grados de Maestro respecto al grado de conocimiento, uso y opinión sobre las herramientas TIC en función de su pertenencia a la titulación de Educación Primaria o Educación Infantil, y a su género, así como al uso de las mismas de acuerdo a sus estilos de aprendizaje.

Tabla 1. Conocimiento sobre herramientas TIC. *Puntuaciones significativas $p<0.05$

\begin{tabular}{|c|c|c|c|c|c|c|}
\hline Conozco: & & $M$ & $D T$ & $F$ & $g l$ & $P$ \\
\hline \multirow{2}{*}{ Programas básicos } & $\operatorname{lnf}$ & 3.62 & .53 & \multirow{2}{*}{1.41} & \multirow{2}{*}{1} & \multirow{2}{*}{.236} \\
\hline & Pri & 3.52 & .57 & & & \\
\hline \multirow{2}{*}{ Redes sociales } & $\operatorname{lnf}$ & 3.74 & .49 & \multirow{2}{*}{5.10} & \multirow{2}{*}{1} & \multirow{2}{*}{$.025^{\star}$} \\
\hline & Pri & 3.56 & .57 & & & \\
\hline \multirow{2}{*}{ Blog, chat, foro } & Inf & 3.43 & .67 & \multirow{2}{*}{10.16} & \multirow{2}{*}{1} & \multirow{2}{*}{$.002^{*}$} \\
\hline & Pri & 3.07 & .73 & & & \\
\hline \multirow{2}{*}{ Portales educativos } & $\operatorname{Inf}$ & 3.23 & .67 & \multirow{2}{*}{.55} & \multirow{2}{*}{1} & \multirow{2}{*}{.458} \\
\hline & Pri & 3.15 & .76 & & & \\
\hline \multirow{2}{*}{ Programas de edición imagen, vídeo y audio } & $\operatorname{lnf}$ & 2.84 & .78 & \multirow{2}{*}{2.60} & \multirow{2}{*}{1} & \multirow{2}{*}{.109} \\
\hline & Pri & 2.63 & .87 & & & \\
\hline \multirow{2}{*}{ Buscadores en la red } & $\operatorname{lnf}$ & 3.51 & .65 & \multirow{2}{*}{1.28} & \multirow{2}{*}{1} & \multirow{2}{*}{.258} \\
\hline & Pri & 3.40 & .66 & & & \\
\hline \multirow{2}{*}{ Traductores online } & $\operatorname{lnf}$ & 3.23 & .72 & \multirow{2}{*}{.74} & \multirow{2}{*}{1} & \multirow{2}{*}{.390} \\
\hline & Pri & 3.13 & .84 & & & \\
\hline \multirow{2}{*}{ Portales de vídeo online } & $\operatorname{lnf}$ & 3.71 & .53 & \multirow{2}{*}{5.18} & \multirow{2}{*}{1} & \\
\hline & Pri & 3.51 & .59 & & & $.024^{\star}$ \\
\hline Riblintoroc virtuoloc & $\operatorname{lnf}$ & 3.30 & .71 & 315 & 1 & 078 \\
\hline Bıbliotecas virtuales & Pri & 3.10 & .70 & 3.15 & 1 & $.0 / 8$ \\
\hline Editoros do nóginos un & Inf & 1.57 & .72 & 00 & 1 & 007 \\
\hline Ealtores de paginas wed & Pri & 1.58 & .74 & .00 & 1 & .981 \\
\hline & $\operatorname{lnf}$ & 3.20 & .79 & & & \\
\hline Navegadores web & Pri & 3.09 & .77 & .80 & 1 & .371 \\
\hline & $\operatorname{lnf}$ & 1.60 & .71 & & & \\
\hline Programas educativos de autor & Pri & 1.79 & .83 & 2.50 & 1 & .116 \\
\hline Actividadoc guiadac do húcgueda on Internot & $\operatorname{lnf}$ & 1.45 & .63 & 485 & 1 & $020 *$ \\
\hline Actıvidades guladas de busqueda en Internet & Pri & 1.72 & .88 & 4.85 & 1 & $.029^{\circ}$ \\
\hline Disnositivos multimedia & $\operatorname{lnf}$ & 3.10 & .79 & & 1 & \\
\hline Dispositivos multımedia & Pri & 2.98 & .86 & .88 & 1 & .349 \\
\hline
\end{tabular}


En relación con el primer bloque, "Conozco herramientas TIC", los estudiantes manifestaron tener un conocimiento alto o muy alto en casi todos los ítems del cuestionario (tabla 1). En 10 de los 14 ítems de este apartado sus puntuaciones medias se situaron por encima de 3 (en una escala máxima de 4) y, más concretamente, en cuatro ítems estas respuestas superaban la puntuación de 3.50 (conozco "Programas básicos", "Redes sociales", "Buscadores en la red" y "Portales de vídeo online"). Por el contrario, los encuestados manifestaron escaso conocimiento sobre editores de páginas web, programas educativos de autor y actividades educativas guiadas en Internet, con medias por debajo de 1.50. Por titulación, en cuatro de estos ítems, se obtuvieron puntuaciones estadísticamente significativas (al $0.5 \%$ o superior), si bien ninguna de las respuestas alcanzó una puntuación muy significativa $(p=.000)$. Así, el alumnado de Educación Infantil mostró tener un mayor conocimiento que el de Primaria en "Redes sociales" ( $M=3.56 ; D T$ $=.57)(p=.025)$; "Blog, chat y foro" $(M=3.43 ; D T=.67)(p=.002) ; \mathrm{y}$ "Portales de vídeo online" $(M=3.71 ; D T$ $=.53)(p=.024)$; mientras que los estudiantes de Primaria, aun teniendo un conocimiento bajo en "Actividades guiadas de búsqueda en Internet", era mejor que el del alumnado de Infantil $(M=1.72 ; D T=.88)$ $(p=.029)$.

Tabla 2. Uso de herramientas TIC. *Puntuaciones significativas $p<0.05$

\begin{tabular}{|c|c|c|c|c|c|c|}
\hline Uso: & & $M$ & $D T$ & $F$ & $g l$ & $P$ \\
\hline \multirow{2}{*}{ Programas básicos } & $\operatorname{lnf}$ & 3.73 & .47 & \multirow{2}{*}{.65} & \multirow{2}{*}{1} & \multirow{2}{*}{.418} \\
\hline & Pri & 3.67 & .54 & & & \\
\hline \multirow{2}{*}{ Redes sociales } & $\operatorname{lnf}$ & 3.63 & .57 & \multirow{2}{*}{3.63} & \multirow[b]{2}{*}{1} & \multirow[b]{2}{*}{.059} \\
\hline & Pri & 3.44 & .68 & & & \\
\hline \multirow{2}{*}{ Blog, chat, foro } & $\operatorname{lnf}$ & 2.48 & .89 & \multirow{2}{*}{1.04} & \multirow{2}{*}{1} & \multirow{2}{*}{.308} \\
\hline & Pri & 2.32 & 1.03 & & & \\
\hline \multirow{2}{*}{ Portales educativos } & $\operatorname{lnf}$ & 3.28 & .69 & \multirow{2}{*}{.62} & \multirow{2}{*}{1} & \multirow{2}{*}{.430} \\
\hline & Pri & 3.37 & .69 & & & \\
\hline \multirow{2}{*}{ Programas de edición imagen, vídeo y audio } & Inf & 2.62 & .79 & \multirow{2}{*}{1.48} & \multirow{2}{*}{1} & \multirow{2}{*}{.225} \\
\hline & Pri & 2.45 & .99 & & & \\
\hline \multirow{2}{*}{ Buscadores en la red } & $\operatorname{lnf}$ & 3.67 & .52 & \multirow{2}{*}{4.02} & \multirow{2}{*}{1} & \multirow{2}{*}{$.046^{*}$} \\
\hline & Pri & 3.48 & .67 & & & \\
\hline \multirow{2}{*}{ Traductores online } & Inf & 3.01 & .76 & \multirow{2}{*}{.83} & \multirow{2}{*}{1} & \multirow{2}{*}{.363} \\
\hline & Pri & 2.90 & .80 & & & \\
\hline \multirow{2}{*}{ Portales de vídeo online } & $\operatorname{lnf}$ & 3.56 & .65 & \multirow{2}{*}{4.89} & \multirow{2}{*}{1} & \\
\hline & Pri & 3.31 & .73 & & & $.028^{\circ}$ \\
\hline Riblinto oc virtu & $\operatorname{lnf}$ & 3.04 & .78 & 63 & 1 & 128 \\
\hline Bidilotecas virtuales & Pri & 2.94 & .79 & .63 & 1 & .428 \\
\hline & Inf & 1.38 & .69 & & & \\
\hline Editores de päginas web & Pri & 1.40 & .62 & .01 & 1 & .906 \\
\hline & Inf & 2.99 & .96 & 137 & & \\
\hline Navegadores web & Pri & 3.16 & .91 & 1.31 & 1 & .242 \\
\hline & $\operatorname{lnf}$ & 1.30 & .51 & & & \\
\hline Programas educativos de autor & Pri & 1.52 & .67 & 5.60 & 1 & $.019^{*}$ \\
\hline & $\operatorname{lnf}$ & 1.32 & .66 & & & \\
\hline Actividades guiadas de busqueda en Internet & Pri & 1.43 & .59 & 1.36 & 1 & .245 \\
\hline Disnocitivos multimedia & $\operatorname{lnf}$ & 2.95 & .85 & 147 & 1 & 226 \\
\hline Dispositıvos multımedia & Pri & 2.78 & .96 & 1.47 & 1 &.$\angle 20$ \\
\hline
\end{tabular}

Respecto al segundo bloque, sobre el "Uso herramientas TIC", los estudiantes manifestaron hacer un uso menor en comparación con el conocimiento que tenían hacia las mismas herramientas (tabla 2). Este hecho quedó patente en que sólo en cuatro de los 14 ítems que componen este subapartado las puntuaciones fueron altas $(M>3.50)$, concretamente en el uso de "Programas básicos", "Redes sociales", "Buscadores en la red" y "Portales de vídeo". Además, los estudiantes expusieron emplear muy poco o casi nada "Editores de páginas web", "Programas educativos de autor" o "Actividades guiadas de búsqueda en Internet". Los estudiantes de Educación Infantil usaban de manera estadísticamente significativa "Buscadores en la red" y "Portales de vídeo online" $(M=3.67 ; D T=.52)(p=.046)$ y $(M=3.56 ; D T=.65)(p=.028)$, respectivamente. Por su lado, los estudiantes de Primaria empleaban escasamente "Programas educativos de autor", si bien lo hacían de una forma superior al mostrado por el alumnado de Infantil ( $M=1.52$ frente a 1.30), con una puntuación estadísticamente significativa de $p=.019$.

Las "Opiniones sobre las herramientas TIC" fueron altamente positivas (tabla 3). Las medias de puntuación más altas $(M>3.50)$ se situaron en los ítems "Ayudan a buscar información", "Son importantes en mi formación académica", "Me ayudan a aprender", "Son imprescindibles hoy en día" y "Son muy útiles para realizar trabajos", si bien dicha opinión arrojó escasas puntuaciones significativas; tan sólo en dos de los ítems de este subapartado del cuestionario. En este sentido, para el alumnado de Infantil, las TIC eran 
consideradas como un "Medio para fomentar las relaciones en clase" $(M=3.04 ; D T=.86)(p=.000)$ muy por encima a la opinión que tenían los estudiantes de Primaria $(M=2.38 ; D T=.88)$. Igualmente, los primeros manifestaron que "No ofrecían seguridad en su privacidad" ( $M=2.12 ; D T=.77)(p=.029)$.

En cuanto al empleo de las herramientas TIC según el estilo de aprendizaje predominante en el alumnado (tabla 4), las puntuaciones medias más altas se situaron en aquellos ítems vinculados con el estilo de aprendizaje pragmático ("Me gusta experimentar con las TIC", "Me gusta aplicar los conocimientos aprendidos con las TIC" y "No me importa emplear TIC para hacer efectivo mi trabajo") y el teórico ("Sé que las TIC son buenas para mi formación"). Si bien, las diferencias entre títulos mostraron puntuaciones estadísticamente significativas en los ítems "Me gusta experimentar con las TIC" (estilo pragmático), "Me gusta el reto de utilizar las TIC" y "Me gusta buscar nuevas experiencias a través de las TIC" (estilo activo), y "Me interesan las opiniones en chat o foros" (estilo reflexivo). De nuevo, los estudiantes de Educación Infantil puntuaron por encima de los de Primaria en estos ítems.

Tabla 3. Opinión sobre las herramientas TIC. *Puntuaciones significativas $p<0.05$

\begin{tabular}{|c|c|c|c|c|c|c|}
\hline Considero que las TIC: & & M & $D T$ & $F$ & $g l$ & $P$ \\
\hline \multirow{2}{*}{ Son importantes en mi formación académica } & Inf & 3.63 & .50 & \multirow{2}{*}{1.20} & \multirow{2}{*}{1} & \multirow{2}{*}{.274} \\
\hline & Pri & 3.54 & .54 & & & \\
\hline \multirow{2}{*}{ Me ayudan a aprender } & Inf & 3.55 & .59 & \multirow{2}{*}{2.16} & \multirow{2}{*}{1} & \multirow{2}{*}{144} \\
\hline & Pri & 3.41 & .58 & & & \\
\hline \multirow{2}{*}{ Perjudican más que ayudan } & Inf & 1.57 & .86 & \multirow{2}{*}{.60} & \multirow{2}{*}{1} & \multirow{2}{*}{.437} \\
\hline & Pri & 1.47 & .74 & & & \\
\hline \multirow{2}{*}{ Son importantes por su aplicación educativa } & Inf & 3.29 & .55 & \multirow{2}{*}{.82} & \multirow{2}{*}{1} & \multirow{2}{*}{.365} \\
\hline & Pri & 3.21 & .60 & & & \\
\hline \multirow{2}{*}{ Ayudan a mejorar mis resultados académicos } & Inf & 3.09 & .72 & \multirow{2}{*}{1.98} & \multirow{2}{*}{1} & \multirow{2}{*}{.161} \\
\hline & Pri & 2.93 & .72 & & & \\
\hline \multirow{2}{*}{ Son un medio para fomentar las relaciones en clase } & Inf & 3.04 & .86 & \multirow{2}{*}{22.61} & \multirow{2}{*}{1} & \multirow{2}{*}{$.000^{*}$} \\
\hline & Pri & 2.38 & .88 & & & \\
\hline \multirow{2}{*}{ Son difíciles de comprender y utilizar } & Inf & 1.66 & .77 & \multirow{2}{*}{.22} & \multirow{2}{*}{1} & \multirow{2}{*}{.637} \\
\hline & Pri & 1.72 & .77 & & & \\
\hline \multirow{2}{*}{ Apoyo para completar conocimientos } & Inf & 3.27 & .62 & 96 & 1 & 327 \\
\hline & Pri & 3.16 & .76 & . & 1 & . \\
\hline Ne froson coguridad on mi nrivacidad & Inf & 2.12 & .77 & 183 & 1 & 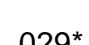 \\
\hline No oirecen seguriaad en mi privaclaad & Pri & 1.85 & .79 & 4.83 & 1 & . \\
\hline Me hogon nordartiomne & Inf & 1.95 & .73 & 235 & 1 & 107 \\
\hline 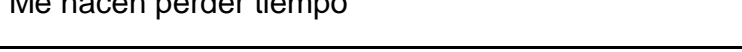 & Pri & 2.16 & .99 & 2.35 & 1 & $.12 r$ \\
\hline No sustituven a recursos educativos tradicionales & Inf & 2.23 & .93 & 29 & 1 & 586 \\
\hline 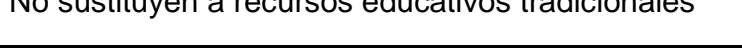 & Pri & 2.31 & .94 & . 29 & & .500 \\
\hline Con imnrocoindiblac houron dín & Inf & 3.48 & .63 & 05 & 1 & $812+2+3$ \\
\hline Som imprescinariones noy en ara & Pri & 3.51 & .69 & . 05 & 1 & . 813 \\
\hline Avudan a buscar información & Inf & 3.78 & .44 & 83 & 1 & 361 \\
\hline . & Pri & 3.72 & .45 & & & \\
\hline Horramionta útil nara trahainc & Inf & 3.68 & .54 & & & \\
\hline Herramienta uil para trabajos & Pri & 3.72 & .53 & .15 & 1 & .694 \\
\hline No crn nonomonto fiobloc on cuinformogión & Inf & 2.21 & .69 & $5 ?$ & 1 & 171 \\
\hline No son plenamente flables en su informacion & Pri & 2.29 & .75 & .52 & 1 & . 411 \\
\hline Sirven para ocio y tiempo libre & Inf & 2.95 & .75 & 23 & 1 & 627 \\
\hline & Pri & 2.89 & .88 & ( 20 & & $.0<r$ \\
\hline
\end{tabular}

Las diferencias significativas en cuanto a género (tabla 5) situaron las valoraciones medias de las estudiantes por encima de las emitidas por el género masculino. Se observan puntuaciones significativas en los ítems: Conozco Programas básicos $(p=.0 .33)$ (Masculino $X=3.32$; Femenino $X=3.6$ ); Conozco Redes sociales $(p=.48)$ (Masculino $X=3.42$; Femenino $X=3.68)$; Uso Programas básicos $(p=.002)$ (Masculino $X=3.37$; Femenino $X=3.74$ ); Uso Redes sociales $(p=.045$ ) (Masculino $X=3.26$; Femenino $X=3.58$ ); Uso Portales educativos $(p=.011$ ) (Masculino $X=2.95$; Femenino $X=3.38$ ); Considero que las TIC son un medio para fomentar las relaciones en clase $(p=.006)$ (Masculino $X=2.16$; Femenino $X=2.78$ ); No me importa emplear TIC para hacer efectivo mi trabajo (pragmático) $(p=.011)$ (Masculino $X=2.74$; Femenino $X=3.21$ ); Analizo pros y contras antes de trabajar con TIC (reflexivo) ( $p=.023$ ) (Masculino $X=1.68$; Femenino $X=3.03$ ). 
Tabla 4. Uso de herramientas TIC de acuerdo con el estilo de aprendizaje predominante. *Puntuaciones significativas $\mathrm{p}<0.05$

\begin{tabular}{|c|c|c|c|c|c|c|}
\hline Uso de las TIC según estilo de aprendizaje: & & $M$ & $D T$ & $F$ & $g l$ & $P$ \\
\hline \multirow{2}{*}{ Me gusta experimentar con las TIC } & Inf & 3.11 & .77 & \multirow{2}{*}{4.56} & \multirow{2}{*}{1} & \multirow{2}{*}{$.034^{*}$} \\
\hline & Pri & 2.84 & .84 & & & \\
\hline \multirow{2}{*}{ Las TIC me ayudan a resolver problemas } & Inf & 2.82 & .83 & \multirow{2}{*}{.04} & \multirow{2}{*}{1} & \multirow{2}{*}{.827} \\
\hline & Pri & 2.79 & .73 & & & \\
\hline \multirow{2}{*}{ Procuro conocer las nuevas TIC } & $\operatorname{lnf}$ & 2.49 & .85 & \multirow{2}{*}{1.21} & \multirow{2}{*}{1} & \multirow{2}{*}{.271} \\
\hline & Pri & 2.35 & .79 & & & \\
\hline \multirow{2}{*}{ Disfruto cuando preparo trabajos con las TIC } & $\operatorname{lnf}$ & 2.88 & .76 & \multirow{2}{*}{1.60} & \multirow{2}{*}{1} & \multirow{2}{*}{.207} \\
\hline & Pri & 2.73 & .77 & & & \\
\hline \multirow{2}{*}{ Sé que las TIC son buenas para mi formación } & Inf & 3.20 & .63 & \multirow{2}{*}{.17} & \multirow{2}{*}{1} & \multirow{2}{*}{.674} \\
\hline & Pri & 3.24 & .64 & & & \\
\hline \multirow{2}{*}{ Me gusta aplicar los conocimientos aprendidos con las TIC } & Inf & 3.15 & .70 & \multirow{2}{*}{.17} & \multirow{2}{*}{1} & \multirow{2}{*}{.674} \\
\hline & Pri & 3.10 & .73 & & & \\
\hline \multirow{2}{*}{ No me importa emplear TIC para hacer efectivo mi trabajo } & Inf & 3.22 & .75 & \multirow{2}{*}{1.23} & \multirow{2}{*}{1} & \multirow{2}{*}{.269} \\
\hline & Pri & 3.09 & .78 & & & \\
\hline \multirow{2}{*}{ Interpreto la información de la Red antes de opinar } & Inf & 3.01 & .65 & & & \\
\hline & Pri & 3.02 & .77 & .01 & 1 & .912 \\
\hline Me austa el reto de utilizar las TIC. & $\operatorname{lnf}$ & 2.65 & .80 & 473 & 1 & $031^{*}$ \\
\hline 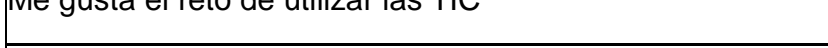 & Pri & 2.37 & .81 & 4.10 & 1 & .001 \\
\hline Me inguieta no noder utilizar lac TIC & Inf & 2.45 & .93 & 317 & 1 & 077 \\
\hline 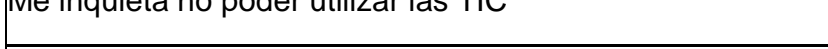 & Pri & 2.20 & .88 & 0.17 & 1 & .017 \\
\hline Me qusta buscar nuevas experiencias a través de las TIC & Inf & 2.79 & .80 & 5.20 & 1 & $.024^{*}$ \\
\hline & Pri & 2.50 & .81 & $0 . \angle 0$ & 1 & \\
\hline Analizo nroc $\mathrm{v}$ contrac antec de trahaiar con TIC & $\operatorname{lnf}$ & 2.16 & .79 & 188 & 1 & 172 \\
\hline Amanzo pros y comiras anies de tranajar com in & Pri & 1.99 & .79 & 1.00 & 1 & .172 \\
\hline Me austa sequir un orden cuando utilizo Internet & Inf & 2.98 & .75 & 04 & 1 & 829 \\
\hline 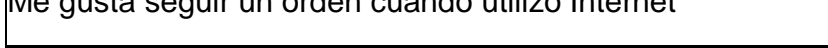 & Pri & 3.00 & .68 & .04 & 1 & $.0<9$ \\
\hline Emoleo la intuición en el uso de las TIC & Inf & 2.30 & .96 & 04 & 1 & 836 \\
\hline & Pri & 2.33 & .77 & & & \\
\hline Me interesan las oniniones en chat o foros & Inf & 2.28 & .87 & 863 & 1 & 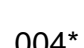 \\
\hline IVie interesan las opiniones en chat o roros & Pri & 1.89 & .82 & 8.03 & 1 & .004 \\
\hline Ohtengn conclucinnec al trahaiar con TIC & Inf & 2.52 & .78 & 54 & 1 & 161 \\
\hline Uotengo conciusiones al tranajar con ill & Pri & 2.62 & .81 & .54 & 1 & .401 \\
\hline
\end{tabular}

Tabla 5. Diferencias estadísticamente significativas en cuanto a género. *Puntuaciones significativas $p<0.05$

\begin{tabular}{|c|c|c|c|c|c|c|}
\hline & & $M$ & $D T$ & $F$ & $g l$ & $P$ \\
\hline \multirow{2}{*}{ Conozco Programas básicos } & Mas & 3.32 & .58 & \multirow{2}{*}{4.63} & \multirow{2}{*}{1} & \multirow{2}{*}{.033} \\
\hline & Fem & 3.60 & .54 & & & \\
\hline \multirow{2}{*}{ Conozco Redes sociales } & Mas & 3.42 & .60 & \multirow{2}{*}{3.96} & \multirow{2}{*}{1} & \multirow{2}{*}{.048} \\
\hline & Fem & 3.68 & .52 & & & \\
\hline \multirow{2}{*}{ Uso Programas básicos } & Mas & 3.37 & .68 & \multirow{2}{*}{9.50} & \multirow{2}{*}{1} & \multirow{2}{*}{.002} \\
\hline & Fem & 3.74 & .46 & & & \\
\hline \multirow{2}{*}{ Uso Redes sociales } & Mas & 3.26 & .65 & \multirow{2}{*}{4.08} & \multirow{2}{*}{1} & \multirow{2}{*}{.045} \\
\hline & Fem & 3.58 & .63 & & & \\
\hline \multirow{2}{*}{ Uso Portales educativos } & Mas & 2.95 & .78 & \multirow{2}{*}{6.66} & \multirow{2}{*}{1} & \multirow{2}{*}{.011} \\
\hline & Fem & 3.38 & .66 & & & \\
\hline \multirow{2}{*}{ Son un medio para fomentar las relaciones en clase } & Mas & 2.16 & .95 & \multirow{2}{*}{7.87} & \multirow{2}{*}{1} & \multirow{2}{*}{.006} \\
\hline & Fem & 2.78 & .91 & & & \\
\hline \multirow{2}{*}{ No me importa emplear TIC para hacer efectivo mi trabajo } & Mas & 2.74 & .87 & \multirow{2}{*}{6.56} & \multirow{2}{*}{1} & \multirow{2}{*}{.011} \\
\hline & Fem & 3.21 & .74 & & & \\
\hline \multirow{2}{*}{ Analizo pros y contras antes de trabajar con TIC } & Mas & 1.68 & .67 & \multirow{2}{*}{5.25} & \multirow{2}{*}{1} & \multirow{2}{*}{.023} \\
\hline & Fem & 3.03 & .72 & & & \\
\hline
\end{tabular}


Conocimiento y Uso de las Tecnologías de la Información y las Comunicaciones (TIC) Cózar-Gutiérrez

\section{DISCUSIÓN}

El análisis de los resultados ofrece unos conocimientos adecuados de las herramientas TIC en cuanto a programas básicos, redes sociales, buscadores en la red y portales de vídeo online. Este resultado evidencia un fuerte uso de las TIC como agentes de comunicación social, ocio y tiempo libre, en comparación con los escasos conocimientos relacionados con editores de páginas web, programas educativos de autor y actividades educativas guiadas en la Red. Por otra parte, es destacable que los grupos de estudiantes pertenecientes al Grado de Maestro en Educación Infantil mostraron un mayor conocimiento en herramientas TIC como redes sociales, blogs, chat y foro y portales de vídeo online que los alumnos pertenecientes a la titulación de Educación Primaria. Detrás de este resultado, podemos observar que hay connotaciones debidas a un predominio del género femenino en los cursos de Infantil, en coincidencia con lo señalado en otras investigaciones en las que se concluye que las mujeres tienden a un uso más social de las TIC (Fernández et al., 2015; Mazman et al, 2011).

En línea con otros autores (Gutiérrez et al., 2010; Roblizo y Cózar, 2015; Roig y Pascual, 2012), se hace un uso menor de las TIC en comparación con el grado de los conocimientos mostrados hacia estas herramientas. En este sentido, el alumnado conocía más que utilizaba programas básicos, redes sociales, buscadores en la Red y portales de vídeo. Este dato puede estar asociado a factores de disponibilidad horaria para indagar y navegar en Internet, hecho que desvela que la utilización de estas herramientas precisa de su propio tiempo y espacio, que hay que restarlo a otras actividades académicas, de estudio y personales.

Resulta preocupante que los estudiantes que participaron en el estudio, futuros maestros, manifestaron un escaso uso de las herramientas TIC con fines educativos, aunque sus opiniones hacia las mismas son altamente positivas, ya que las consideran como una importante ayuda para la formación académica, la realización de trabajos y para un correcto desenvolvimiento en la sociedad actual. Prendes, Castañeda y Gutiérrez (2010) inciden en lo generalizado de estos mediocres resultados en buena parte de los estudios realizados sobre estudiantes de magisterio. Esta respuesta, en apariencia contradictoria, puede estar condicionada por la carencia de una asignatura específica en el plan de estudios, en esta etapa universitaria destinada a la formación inicial, donde se contemple y estudie la aplicación educativa de las TIC.

En la incidencia de la variable género, los resultados de esta investigación no coinciden con los estudios que hablan de la existencia de una segundo y tercera brecha digital de género (Castaño, 2008; Castaño, et al., 2011; Vázquez y Castaño, 2011), sino que se indica una tendencia inversa. Las puntuaciones de las mujeres están por encima en la mayor parte de los ítems e incluso destacan en nueve de ellos con diferencias estadísticamente significativas frente a los hombres. Estos datos coinciden con lo señalado en otros trabajos en los que se muestra que con la llegada de la Web 2.0 y las redes sociales, la brecha digital tiende a igualarse (Clipson et al, 2012) e incluso a invertirse (Vico et al., 2015).

En cuanto a la vinculación de las TIC con el estilo de aprendizaje predominante, las respuestas más altas vinculadas con los ítems relacionados con el estilo pragmático y teórico, parecen indicar que se debe a la doble vertiente propia de las TIC. Por un lado, son recursos manipulativos (así se relacionarían con el estilo pragmático) y por otra parte, son medios intuitivos (algo que se vincularía con el estilo teórico). Este resultado pone de manifiesto la necesidad de incidir en el trabajo de actividades con las TIC que combinen ambas posibilidades, la manipulación y la intuición, con la finalidad de reforzar los citados estilos de aprendizaje. En este aspecto, de nuevo se observaron mejores puntuaciones entre el alumnado del Grado de Maestro en Educación Infantil, posiblemente asociado a un rasgo psicológico de mayor intuición afectiva que se muestra con más fuerza en el género femenino que en el masculino (Gigerenzer et al., 2014). Y ya hemos indicado que en esta titulación predomina el número de alumnas.

Por otra parte, el hecho de que no se observara un estilo de aprendizaje predominantemente claro en el uso y empleo de las TIC muestra la complejidad de los estilos de aprendizaje (la individualidad de los procesos cognitivos) y las distintas formas de usar las TIC, de tal modo que se puede afirmar que existen tantas formas de aprender y utilizar las TIC como personas hay (la individualidad en la formación tecnológica y virtual) (Cózar et al., 2015; De Moya et al., 2011; García-Cué et al., 2004). Por este motivo, los estilos de aprendizaje y el conocimiento y uso de las herramientas TIC se establecen como formas y medios de aprendizaje personal asociados a las características particulares de cada individuo.

En el ámbito universitario, en los últimos años se ha constatado un creciente interés por conocer la forma particular en la que el alumnado realiza su propio aprendizaje, en el marco de una enseñanza más significativa y ajustada a las necesidades de aprendizaje de cada estudiante (García-Cué et al., 2004; Gundín y Fernández-Martínez, 2005). Esta nueva realidad en los contextos educativos constituye un campo de estudio de primer orden, al que ha ido paralelo la irrupción de una conciencia crítica en las aulas 
universitarias acerca de la conveniencia de diseñar actividades ajustadas a las particularidades individuales de aprendizaje (Alonso y Gallego, 2003). Detrás de esta evidencia subyace la superación del modelo clásico expositivo en aras de una renovación pedagógica que dé respuesta a los retos formativos derivados de las demandas de la sociedad actual.

Si queremos ofrecer una educación de calidad, es preciso contribuir a la madurez personal del alumnado, para que adquiera seguridad personal, con actitud positiva hacia los retos, sin temor a dificultades, y dispuestos a conseguir el éxito en las tareas que emprendan. En este sentido, puede ser de gran ayuda que los alumnos se conozcan a sí mismos, siendo un buen medio la aplicación de los estilos de aprendizaje como punto de partida. Para ello, consideramos conveniente que tengan un conocimiento mínimo acerca de lo que son, lo que significan, cuál es el estilo predominante personal y qué estrategias se pueden aplicar para fortalecer puntos débiles y lograr una mejora global de la persona. $\mathrm{Y}$ todo ello, inserto en una formación donde se vincule con el uso y valoración de las TIC, tanto en su formación universitaria como en su faceta más cotidiana y personal, de ocio y relación social. Así, estaremos potenciando el desarrollo de las competencias necesarias para una correcta inclusión en la sociedad el día de mañana, como agentes laborales y ciudadanos activos y participativos con el entorno.

La cultura tecnológica, que ha calado en todos los procesos educativos en un corto espacio de tiempo, condiciona la forma de enseñar y aprender, así como las relaciones interpersonales entre los miembros de la comunidad educativa. Las posibilidades que brindan las TIC hoy en día a través de herramientas didácticas, recursos digitales y entornos virtuales de aprendizaje, permiten la creación o selección de actividades específicas, más motivadoras y personalizadas, de acuerdo con las diferentes formas de aprender. Ser conscientes de este hecho contribuirá, sin duda, a mejorar la calidad de enseñanza en el marco de la educación superior, facilitando al alumnado nuevas posibilidades formativas y un enriquecimiento personal.

Las TIC no son un remedio milagroso para lograr una educación de calidad, ya que no dejan de ser un medio para lograr este fin. Sin embargo, las aulas del siglo XXI ya no se conciben sin la aplicación de la didáctica digital, pero por sí mismas no dan respuesta a los interrogantes que surgen en el camino de la búsqueda del conocimiento. Profesores y alumnos, auténticos protagonistas del proceso educativo, deben esforzarse por encontrar una optimización de todos los recursos que nos oferta la tecnología y saber adecuarlos a las necesidades específicas de cada materia e, incluso, de cada persona. Para esta tarea, es una excelente ayuda conocer los estilos de aprendizaje.

La entrada de las TIC en los campus universitarios, Facultades y Escuelas Superiores, incorporan un esfuerzo nuevo a los docentes: ya no basta con saber manejar estas nuevas tecnologías y su aplicación en el área de conocimiento específica de cada uno, sino que, además, obligan a descubrir el uso y la actitud de sus alumnos hacia las mismas, si quieren triunfar en el empeño de lograr un correcto aprendizaje. Este objetivo motiva nuevas investigaciones que relacionen docencia y TIC y es el motor que nos ha llevado a realizar la investigación que presentamos.

Es conveniente plantear una limitación a la presente investigación. Al tratarse de una muestra reducida y no representativa de la población universitaria española, los resultados no se pueden extrapolar a otros contextos más generales, pero se pueden comparar con otros estudios de corte similar. No obstante, la misma se convierte en un avance de líneas de investigación futuras, pues consideramos interesante seguir investigando en la relación existente entre el alumno universitario y las nuevas tecnologías, en aspectos tales como los ya dichos a los que podemos añadir otros como el interés que sienten hacia descubrir nuevos aspectos y utilidades de las TIC, sobre todo, como herramienta pedagógica en su formación inicial como maestros (en nuestro caso), en las diversas áreas formativas y su grado de aceptación y valoración como herramientas de aprendizaje en el aula universitaria.

\section{CONCLUSIONES}

A partir del análisis de los resultados obtenidos y de la discusión planteada se puede concluir de un modo estructurado:

a) Existe un conocimiento notable y una opinión favorable sobre las TIC por parte del alumnado participante en la investigación, un resultado que contrasta con el uso que hacen de las mismas, que fue ligeramente menor al esperado.

b) Por grupos, la valoración más positiva de los estudiantes del Grado de Infantil en cuanto al conocimiento y uso de las TIC ha evidenciado que las herramientas digitales han cobrado una mayor relevancia para su formación, en contraposición con el Grado de Primaria en el que dichas herramientas se consideraban a 
priori más generalizadas. Las mujeres puntuaron por encima de las valoraciones del género masculino, especialmente en relación con el uso de las redes sociales, por lo que no se percibe brecha digital de género.

c) Se observa un estilo de aprendizaje ligeramente predominante en el empleo de las TIC; sobresale un estilo vinculado a saberes prácticos (pragmático) por encima del teórico; un resultado esperable ya que las TIC movilizan unas habilidades más manipulativas.

Finalmente, insistimos en la idea principal ya planteada al inicio de este artículo, que es positivo conocer el grado de comprensión, conocimiento y utilidad que hace el alumnado universitario de las TIC en su vida cotidiana. Es preciso ayudar al alumnado para que sean competentes en el mundo digital en todo aquello referido con su actividad académica, puesto que la oferta y utilidad que ofrecen las nuevas tecnologías es mucho más amplia que el uso más extendido y practicado entre los jóvenes, el cual se desarrolla, principalmente, en ámbitos cotidianos como son los de las redes sociales, lúdicos y de ocio. La ciudadanía del futuro reclama individuos conocedores, debidamente formados y capacitados para un buen y acertado uso de las TIC, tanto en lo personal, como en lo académico, laboral, social y de disfrute.

\section{REFERENCIAS}

Acevedo, D., Cavadia, S. y Alvis, A. Estilos de Aprendizaje de los Estudiantes de la Facultad de Ingeniería de la Universidad de Cartagena (Colombia). doi: 10.4067/S0718-50062015000400003. Formación Universitaria, 8(4), 15-22 (2015)

American Educational Research Association, Ethical standars of the American Educational Research Association. Washington, American Educational Research Association, (en la web: http://www.aera.net/About-AERA/AERA-Rules-Policies/Professional-Ethics, acceso: 14 de mayo 2014) (2011)

Alducin-Ochoa, J.M. y Vázquez-Martínez, A. Autoevaluación de Conocimientos Previos y Rendimiento según Estilos de Aprendizaje en un Grado Universitario de Edificación. doi: 10.4067/S071850062016000200004. Formación Universitaria, 9(2), 29-40 (2016)

Alonso, C. M., Gallego, D.J. y Honey, P., Los estilos de aprendizaje. Procedimientos de diagnóstico y mejora. Ediciones Mensajero, Bilbao (1994)

Alonso, C. M. y Gallego, D.J., Cómo diagnosticar y mejorar los estilos de aprendizaje. UNED, Madrid, (2003)

Blumen, S., Rivero, C. y Guerrero, D., Universitarios en educación a distancia: estilos de aprendizaje y rendimiento académico. Revista de Psicología: 29(2), 225-243 (2011)

Cabero, J., Formación del profesorado universitario en TIC. Aplicación del método Delphi para la selección de los contenidos formativos, doi: 10.5944/educxx1.17.1.10707, Educación XX1, 17(1), 111-132 (2014)

Calvache, L., Gómez, M.R. y Poveda, O. Relación entre los estilos de aprendizaje y el uso de las TIC en el proceso de aprendizaje de los estudiantes universitarios, http://www.cide.edu.co/ojs/index.php/ silogismo/index; ISSN: 1909-955X, Silogismo. Revista de Investigación, 11, 21-43 (2013)

Castejón, J.L. Introducción a los métodos y técnicas de investigación y obtención de datos en psicología. Alicante: ECU (1997)

Castaño, C. Nuevas tecnologías y género. La segunda brecha digital y las mujeres. Telos: Cuadernos de comunicación e innovación, 75, 24-33 (2008)

Castaño, C., Martín, J. y Martínez, J.L. La brecha digital de género en España y Europa: medición con indicadores compuestos, doi: 10.5477/cis/reis.136.127, Reis. Revista Española de Investigaciones Sociológicas, 136, 127-140 (2011)

Clipson, T. W., Wilson, A. y DuFrene, D. D. The Social Networking Arena: Battle of the Sexes, doi: 10.1177/1080569911423961, Business and Professional Communication Quarterly, 75(1), 64-67 (2012)

Cózar, R., De Moya, M.V., Hernández, J.A. y Hernández, J.R. TIC, estilos de aprendizaje y competencia musical en los estudios de Grado de Maestro, doi: 10.5209/rev_RECIEM.2015.v12.47752, Revista Electrónica Complutense de Investigación en Educación Musical, 12, 73-85 (2015) 
Cózar, R. y De Moya, Mạ. V. (Coords.), Las TIC en el aula desde un enfoque multidisciplinar. Aplicaciones prácticas. Octaedro, Barcelona (2013)

Cózar, R. y Roblizo, M., La competencia digital en la formación de los futuros maestros. Percepciones de los alumnos de los Grados de Maestro de la Facultad de Educación de Albacete, doi: 10.17398/1695288X.13.2.119, RELATEC. Revista Latinoamericana de Tecnología Educativa: 13 (2), 119-133 (2014)

De Moya, M.B., Hernández, J.R., Hernández, J.A. y Cózar, R., Análisis de los estilos de aprendizaje y las TIC en la formación personal del alumnado universitario a través del cuestionario REATIC, RIE. Revista de Investigación Educativa: 29 (1), 137-156 (2011)

Evans, C., Cools, E. y Charlesworth, Z. M., Learning in higher education: How cognitive and learning styles matter, doi: 10.1080/13562517.2010.493353, Teaching in Higher Education: 15 (4), 467-478 (2010)

Fernández, J., Peñalba, A. e Irazabal, I. Hábitos de uso y conductas de riesgo en Internet en la preadolescencia. doi: 10.3916/C44-2015-12 Comunicar, 44, 113-120 (2015)

Galanouli, D., Murphy, C. y Gardner, J. Teachers'perception of the effectiveness of ICT-competence training, doi: 10.1016/j.compedu.2003.12.005, Computers and Education, 43, 63-79 (2004)

Gallego, M.J., Gámiz, V. y Gutiérrez, E., El futuro docente ante las competencias en el uso de las tecnologías de la información y comunicación para enseñar, doi: 10.21556/edutec.2010.34.418, EDUTEC. Revista electrónica de tecnología educativa, 34, 1-18 (2010)

García-Cué, J. L., Santizo, J. A. y Jiménez, M., Identificación de la tecnología computacional de profesores de postgrado de acuerdo a sus estilos de aprendizaje, Actas I Congreso de Estilos de Aprendizaje, Anaya, Madrid (2004)

García-Cué, J. L., Santizo, J. A. y Alonso, C. M., Uso de las TIC de acuerdo a los estilos de aprendizaje de docentes y discentes, Revista Iberoamericana de Educación: 48 (2), 1-14 (2009)

García Retana, J. A., Reflexiones sobre los estilos de aprendizaje y el aprendizaje del cálculo para Ingeniería, doi: 10.15517/aie.v13i1.11717, Revista Actualidades Investigativas en Educación: 13 (1), 124$132(2013)$

García, L., Escalante, L., Fernández, L.G., Escandón, M.C., Mustri, A. y Puga, I., Procesos de enseñanza aprendizaje. Documento de trabajo SEP. Dirección General de Investigación Educativa de la SEP, Fondo Mixto de Cooperación Técnica y Científica México-España, (2000)

Gargallo, B.; Suárez, J. y Ferreras, A., Estrategias de aprendizaje y rendimiento académico en estudiantes universitarios. RIE. Revista de Investigación Educativa: 25 (2), $421-441$ (2007)

Gigerenzer, G., Galesic, M. y Garcia-Retamero, R., Stereotypes About Men's and Women's Intuitions: A Study of Two Nations, doi: 10.1177/0022022113487074, Journal of Cross-Cultural Psychology: 45 (1), $62-81$ (2014)

González, D., Castañeda, S. y Maytorena M. A., Estilos de aprendizaje y aprovechamiento en ingresantes universitarios. Revista de Psicología, 18 (2), 199-225 (2000)

Gundín, O. y Fernández-Martínez, M., Formación del profesorado y estilos de aprendizaje: revisión de estudios empíricos internacionales, Comunicación y Pedagogía: 195, 78-82 (2005)

Gutiérrez, A. Las TIC en la formación del maestro. «Realfabetización» digital del profesorado, Revista Interuniversitaria de Formación de Profesorado, 22(3), 191-206 (2008)

Gutiérrez, A., Palacios, A. y Torrego, L., La formación de los futuros maestros y la integración de las TIC en la educación: anatomía de un desencuentro. Revista de Educación, 352, 267-293 (2010)

Herrera, A. M., Una mirada reflexiva sobre las TIC en Educación Superior, http://redie.uabc.mx, ISSN: 16074041, Revista Electrónica de Investigación Educativa: 17 (1), 1-4 (2015)

Juárez, C.S., Rodríguez, G. y Luna, E. El cuestionario de estilos de aprendizaje CHAEA y la escala de estrategias de aprendizaje ACRA como herramienta potencial para la tutoría académica, http://learningstyles.uvu.edu/index.php/jls, ISSN: 2332-8533, Revista Estilos de Aprendizaje, 10, 1-31 (2012) 
Kirschner, P. y Davis, N., Pedagogic benchmarks for information and communications technology in teacher education. DOI: 10.1080/14759390300200149, Technology, Pedagogy and Education, 12(1), 125-147 (2003)

Koehler, J. y Mishra, P., Introducing Technological Pedagogical Knowledge. AACTE (Eds.). The Handbook of Technological Pedagogical Content Knowledge for Educators. Routledge/Taylor \& Francis Group/American Association of Colleges of Teacher Education (2008)

Komarraju, M., Karau, S. J., Schmeck, R. R. y Avdic, A., The Big Five personality traits, learning styles and academic achievement, doi: 10.1016/j.paid.2011.04.019, Personality and Individual Differences: 51, $472-477$ (2011)

Llorente, M.C., Aspectos fundamentales de la formación del profesorado en TIC, http://acdc.sav.us.es/ojs/index.php/pixelbit/index, ISSN: 2171-7966, Pixel-Bit. Revista de Medios y Educación, 31, 121-130 (2008)

Mazman, G. y Usluel, Y. Gender differences in using social networks, HTTP://www.tojet.net, ISSN: 21467242, The Turkish Online Journal of Educational Technology, 10(2), 133-139 (2011)

Mcvee, M. Bailey, N. y Shanahan, L., Teachers and teacher educators learning from new literacies and new technologies, DOI: 10.1080/10476210802250216, Teaching Education, 19(3), 197-210 (2008)

Monereo, C., Estrategias de enseñanza y aprendizaje. Formación del profesorado y aplicación en la escuela, Grao, Barcelona (2007)

Morón, R., Cardoso, E., Cerecedo, M.T. y Ortíz, J. Evaluación de las Competencias Docentes de Profesores Formados en Instituciones de Educación Superior: El Caso de la Asignatura de Tecnología en la Enseñanza Secundaria, doi: 10.4067/S0718-50062015000300007, Formación Universitaria, 8(3), 57-64, (2015)

Pantoja, M. A., Duque, L. I. y Correa, J. S., Modelos de estilos de aprendizaje: una actualización para su revisión y análisis, doi: 10.17227/01203916.64rce79.105, Revista Colombiana de Educación: 64 (1), 79-105 (2013)

Peterson, E. R., Rayner, S. G y Armstrong, S. J., Researching the psychology of cognitive style and learning style: Is there really a future?, doi: 10.1016/j.lindif.2009.06.003, Learning and Individual Differences: 19, 518523 (2009)

Prendes, M.P., Castañeda, L. y Gutiérrez, I., Competencias para el uso de TIC de los futuros maestros, doi: 10.3916/C35-2010-03-11, Comunicar, 35(18), 175-182 (2010)

Rangel, A.: Competencias docentes digitales: propuesta de un perfil, doi: 10.12795/pixelbit.2015.i46.15, Pixel-Bit. Revista de Medios y educación, 46, 239-241 (2015)

Raposo, M., Fuentes, E. y González, M., Desarrollo de competencias tecnológicas en la formación inicial de maestros., http://relatec.unex.es, ISSN: 1695-288x, RELATEC. Revista Latinoamericana de Tecnología Educativa, 5(2), 525-537 (2006)

Roblizo, M. y Cózar, R., Usos y competencias en TIC en los futuros maestros de Educación Infantil y Primaria: hacia una alfabetización tecnológica real para docentes, doi: 10.12795/pixelbit.2015.i47.02, Pixel Bit, Revista de Medios y Educación: 47, 23-39 (2015)

Roblizo, M., Sánchez, M.C. y Cózar, R., El reto de la competencia digital en los futuros docentes de Infantil, Primaria y Secundaria: los estudiantes de Grado y Máster de Educación ante las TIC, http://www.isdfundacion.org/publicaciones/revista/index.html, ISSN: 1989-3469, Prisma Social: 15 (2015)

Rodríguez, J., Validación del CHAEA en estudiantes universitarios, (en la web: www.circle.adventist.org/files/download/Validchaea.pdf, acceso: 2 de julio 2012) (2006)

Roig, R. y Pascual, A.M. Las competencias digitales de los futuros docentes. Un análisis con estudiantes de Magisterio de Educación Infantil de la Universidad de Alicante. Doi: 10.7203/attic.9.1958, @tic. Revista d'innovació educativa. 9, 53-60 (2012)

Silva, J., Gros, B., Garrido, J.M. y Rodríguez, J., Estándares en tecnologías de la información y la comunicación para la formación inicial docente: situación actual y el caso chileno. Revista Iberoamericana de Educación, 38(3), 1-16 (2006) 
Simón, V. Diversidad de estilos de aprendizaje en el aula de Música de ESO. http://www.uclm.es/ab/educacion/ensayos, ISSN: 2171-9098, Ensayos. Revista de la Facultad de Educación de Albacete, 26, 179-195 (2011)

Tello, J. y Aguaded, I., Desarrollo profesional docente ante los nuevos retos de las tecnologías de la información y la comunicación en los centros educativos. http://acdc.sav.us.es/ojs/index.php/pixelbit/index, ISSN: 2171-7966, Pixel Bit, Revista de Medios y Educación, 34, 31-47 (2009)

Tondeur, J., Van Braak, J., y Valcke, M. Curricula and the use of ICT in education. Two worlds apart? doi:10.1111/i.1467-8535.2006.00680.x British Journal of Educational Technology, 38 (6), $962-975$ (2007)

Van Braak, J., Tondeur, J. y Valcke, M. Explaining types of computer use among primary school teachers. doi:10.1007/BF03173218 European Journal of Psychology of Education, 14 (4), 407-422 (2004)

Varela, L. y Novo, I. Influence of cultural conditions on the use of ICT for learning: differences among Erasmus an national students at Spanish university, doi: 10.4018/IJKSR.2014100104, International Journal of Knowledge Society Research, 5(4), 35-44 (2014)

Vázquez, S. y Castaño, C., La brecha digital de género: prácticas de e-inclusión y razones de la exclusión de las mujeres, http://www.e-revistes.uji.es/index.php/asparkia/index, ISSN: 2340-4795, Asparkía, 22, 33-49 (2011)

Ventura, A.C. El ajuste instructivo entre estilos de aprendizaje y enseñanza en la universidad. Revista de Psicología, 31(2), 266-286 (2013)

Vico, A., Mayor, V. y Rebollo, A. El género y las actitudes hacia las TIC como factores predictores de las competencias digitales en las redes sociales. XXIII Jornadas Universitarias de Tecnología Educativa, (en la web: http://eventos.unex.es/file manager/get paper/2222, acceso: 2 de marzo 2016) (2015)

Yuen, A.H.K. y Ma, W.W.K. Exploring teacher acceptance of e-learning technology, doi: 10.1080/13598660802232779, Asia-Pacific Journal of Teacher Education, 36(3), 229-243 (2008) 